\title{
Reconstitution in cultured cells of replicating HDV RNA from pairs of less than full-length RNAs
}

\author{
SEVERIN O. GUDIMA, JINHONG CHANG, and JOHN M. TAYLOR
}

Fox Chase Cancer Center, Philadelphia, Pennsylvania 19111, USA

\begin{abstract}
The genome of hepatitis delta virus (HDV) is a small single-stranded circular RNA that is replicated via RNA-directed RNA synthesis. This makes use of a host RNA polymerase, probably pol II, that normally transcribes DNA templates. In vivo, the host polymerase can initiate replication from transfected linear RNAs using intramolecular template-switching. The present studies report that the polymerase could also achieve intermolecular switching leading to "reconstitution" of full-length HDV RNAs following transfection with two linear RNAs that were less than full length and yet lacking different regions of the genome. These two RNAs were synthesized in vitro, gel purified, pre-annealed, and then transfected into 8293 , a cell line conditionally expressing the small delta antigen that is essential for HDV replication. Northern analyses of total RNA harvested from transfected cells detected the accumulation of full-length HDV genomic and antigenomic RNAs. Such reconstitution of fulllength replicating HDV RNA was also achieved using nine other pairs of antigenomic RNAs and three pairs of genomic RNAs. Annealing of the RNAs prior to transfection was required for detectable HDV reconstitution. A second cell line, Huh7, also supported reconstitution when a pair of RNAs was cotransfected together with mRNA for the small delta protein. Taken together, these results support a model that observed genome reconstitution is a special form of recombination involving intermolecular template switches and they provide insights into the mechanism of RNA-directed RNA transcription catalyzed by a host RNA polymerase.
\end{abstract}

Keywords: hepatitis delta virus; RNA-directed transcription; RNA polymerase II; template-switching; reconstitution; recombination

\section{INTRODUCTION}

There are many precedents for inter- and intramolecular template-switching by viral polymerases. For example, with retroviruses and hepatitis B viruses, intramolecular switching is part of the normal cycle of genome replication (Flint et al. 2004). Intermolecular template-switching recombination events frequently occur between replicating viral RNA genomes (Lai 1992; Nagy and Simon 1997; Chetverin 1999; Worobey and Holmes 1999). Examples include many positive-strand RNA viruses and a few negative-strand viruses (Chare et al. 2003; Spann et al. 2003).

Most RNA viruses encode an RNA polymerase that is used for (1) replication, (2) template-switching, and in some cases, (3) recombination. However, our attention is focused on human hepatitis delta virus (HDV), an agent

Reprint requests to: John M. Taylor, Fox Chase Cancer Center, 333 Cottman Avenue, Philadelphia, PA 19111-2497, USA; e-mail: John. Taylor@FCCC.edu; fax: (215) 728-3105.

Article published online ahead of print. Article and publication date are at http://www.rnajournal.org/cgi/doi/10.1261/rna.7164905. with a small 1679-nt single-stranded circular RNA genome that is replicated by a form of RNA-directed RNA synthesis that is unique because it is via the redirection of a host RNA polymerase (Gerin et al. 2001; Taylor 2003). HDV replication produces the antigenome, an exact complement of the genome, along with relatively lower amounts of an 800-ntlong capped and polyadenylated RNA that acts as the mRNA for translation of the small delta protein $(\delta \mathrm{Ag})$, a 195 amino acid species essential for HDV genome replication (Chen et al. 1986; Chao et al. 1990).

Much evidence supports the interpretation that the HDV genome is replicated by the host RNA polymerase II, an enzyme that is normally involved in DNA-directed host RNA synthesis (Modahl et al. 2000; Moraleda and Taylor 2001; Macnaughton et al. 2002; Taylor 2003). Furthermore, when HDV replication is initiated from linear RNA templates, this host polymerase achieves intramolecular template-switching (Chang and Taylor 2002; Gudima et al. 2004). In some cases, initiation can be successful despite the apparent need for two intramolecular template switches (Gudima et al. 2004). Given this success, the following stud- 
ies were undertaken to determine whether it was possible to obtain in vivo, intermolecular template-switching between two different HDV RNA templates.

Our strategy was to transfect cells with two different HDV RNAs, each of which was less than full length, but such that by template-switching, it was theoretically possible to reconstitute a replication-competent full-length RNA. This assay for intermolecular template-switching was expected to be infinitely more sensitive than one for recombination between two replication-competent RNAs or even between one replication-competent RNA and a donor RNA fragment, since neither RNA, by itself, has the ability to achieve replication.

Precedents for such intermolecular switching have been reported for other RNA viral systems. In virtually all cases such reconstitution has depended on the initial provision of the viral RNA polymerase (Bergmann et al. 1992; Koetzner et al. 1992; Liao and Lai 1992; Gallei et al. 2004). In most of these studies it has been accepted that the process is mediated via template switches during RNA-directed RNA synthesis. What is more interesting in the case of HDV is that the polymerase used is not a viral enzyme but rather a host RNA polymerase whose normal function is in DNA-directed transcription.

With our strategy we achieved within transfected cells a reconstitution of replicating full-length HDV RNA. Several factors that might affect this phenomenon were investigated. These results are discussed from the perspective of understanding of the enzymatic capabilities of host RNA pol II, and the possibility of recombination during HDV replication.

\section{RESULTS}

\section{Assay for reconstitution}

For our initial efforts to achieve reconstitution of replicating HDV RNA a pair of less than full-length antigenomic RNAs were used. These RNAs were transfected into cells that provided in trans the small delta protein which is essential for genome replication (Chao et al. 1990). Figure 1A is an example of this strategy. Consider first the representation of the full-length antigenomic RNA shown as RNA 0. We used this linear form, as transcribed in vitro, rather than the circular form, as produced in vivo, during HDV replication. We considered that such a linear RNA, which requires intramolecular template-switching to achieve initiation of replication, was a more appropriate positive control for the reconstitution by intermolecular template-switching using less than unit-length RNAs that were also linear.

This unit-length RNA 0 is shown in Figure $1 \mathrm{~A}$ as collapsed into a rodlike structure. This structure is predicted to be the conformation of both genomic and antigenomic HDV RNAs. The evidence for this includes (1) the prediction from the nucleotide sequence of extensive $(74 \%)$ in-
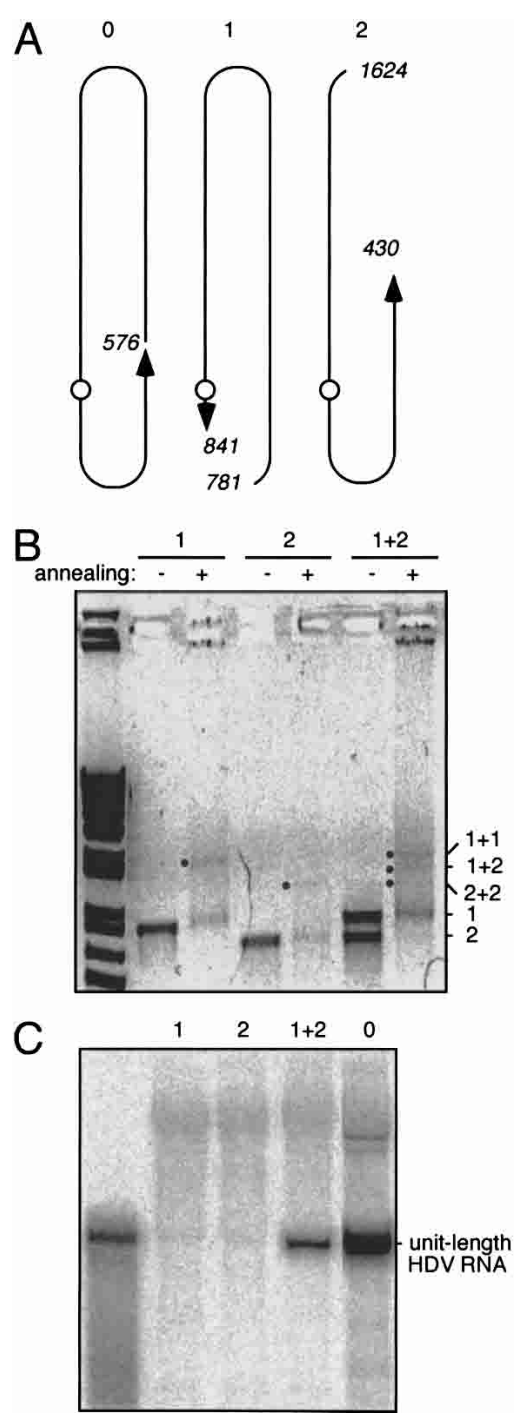

FIGURE 1. Strategy for testing HDV genome reconstitution. (A) Representations of three forms of HDV antigenomic RNA. RNA 0 is unit length and linear. It is drawn to indicate intramolecular folding into an unbranched rodlike structure. The opening site on the 1679-nt-long sequence is indicated using the numbering of Kuo et al. (1989). RNAs 1 and 2 are less than unit-length antigenomic RNAs, with the positions of the $5^{\prime}$ and $3^{\prime}$ ends as indicated. The site of cleavage for the antigenomic ribozyme is indicated by a circle. $(B)$ Nondenaturing agarose gel electrophoresis of RNA species 1,2 , and a mixture of $1+2$, both before and after a prior annealing. RNA in the gel was detected by staining with ethidium bromide followed by photography with reversal of the image. Indicated by dots and also by labels at the right side are the RNA pairs $1+1,1+2$, and $2+2$, that arise because of the annealing. The left lane is a marker of double-stranded DNA species. (C) Northern analysis of the total RNA extracted from $\delta 293$ cells at 4 $\mathrm{d}$ after transfection with pre-annealed RNAs, as indicated. The left lane is a standard of $1.1 \times$ unit-length HDV RNA transcribed in vitro. The assay detects the formation of unit-length genomic RNA arising following the transfection with the antigenomic RNAs.

tramolecular base pairing, (2) the electron microscopic detection of a denaturable rodlike structure, and (3) the electrophoretic mobility of the RNA (Chen et al. 1986; Kos et al. 1986; Wang et al. 1986; Lazinski and Taylor 1994). 
RNAs 1 and 2 of Figure 1A are a representation of the two less than full-length RNAs that were used initially as partners for the reconstitution. Note that there are regions on these two RNAs that theoretically have the ability to interact and make an intermolecular association. This is because they can join to form subregions of the rodlike structure, with the expected $74 \%$ base pairing. In addition, note that the two RNAs have other regions, located at the $3^{\prime}$ and $5^{\prime}$ ends that are exactly repeated. (These correspond to $1624-841=783$ and $781-430=351$ nt.) Therefore, we would expect that if an intermolecular structure is achieved, these repeat sequences might provide additional opportunities for template-switching. This presumption is based on our interpretation of previous studies where greater than unit-length RNA templates were several times more efficient at initiating HDV replication than unit-length RNAs (Gudima et al. 2004).

Figure 1B shows ethidium bromide staining following nondenaturing gel electrophoresis of the two RNAs before and after they were subjected to prior annealing. For both RNAs 1 and 2, after the annealing, we detected a residue of the monomers plus a new band that we interpret as dimers and a broad region of stained material consistent with being heterogeneous high molecular weight aggregates. In addition, when equal moles of the two RNAs were mixed, the annealing led to formation of what we interpret as heterodimers as well as homodimers and higher molecular weight structures.

These annealed RNA species were then transfected into $\delta 293$ cells (Gudima et al. 2004; J. Chang, S.O. Gudima, C. Tarn, X. Nie, and J.M. Taylor, in prep.). These cells contain a single integrated copy of a cDNA for $\delta \mathrm{Ag}$, the expression of which is induced by the addition of tetracycline. In the absence of tetracycline these cells make low amounts of $\delta \mathrm{Ag}$ but the level is enhanced to about 2 million copies per cell soon after the antibiotic is added (J. Chang, S.O. Gudima, C. Tarn, X. Nie, and J.M. Taylor, in prep.). At $1 \mathrm{~d}$ after transfection with the HDV RNAs, aliquots of the cells were reseeded in the presence of tetracycline. Such aliquots were harvested at days 2, 4, and 6. Total RNA was extracted and examined by Northern to detect genomic HDV RNA sequences, with results for day 4 as shown in Figure 1C. No HDV-specific band was detected when cells were transfected individually with either RNA 1 or RNA 2. In contrast, a single band of HDV genomic RNA was detected in cells transfected with the pre-annealed mixture of RNAs 1 and 2. The same band was obtained in cells transfected with full-length linear antigenomic RNA, indicated as RNA 0. Relative to the $1.1 \times$ unit-length RNA standard shown in the left lane, this band was of a mobility expected for full-length genomic RNA. In addition, using a separate analysis of these samples, we were able to detect the fulllength RNA that was antigenomic (data not shown). (Similar results were obtained for RNA extracted at days 2 and 6 after transfection [data not shown].)
These initial positive results support the hypothesis that template-switching during the transcription of the two less than full-length RNAs leads to greater than unit-length nascent transcripts that can be processed to produce replication-competent full-length HDV RNA. If this is correct, the apparent efficiency was about $5 \%$ relative to the level of replication achieved with a single full-length RNA. As will now be described, further experiments were designed to test this interpretation and to explore the influence of certain factors.

\section{Factors affecting reconstitution}

The following studies were undertaken to help resolve factors that might influence the ability to achieve reconstitution. We began by examining the possible role of the primary and secondary structures of the RNA templates. For this purpose we designed 10 additional antigenomic RNAs, indicated in Figure 2 as RNAs 3 to 12. It can be seen that these RNAs vary in length, sequence representation, and the extent of predicted rodlike folding. We also designed four RNAs that were of genomic rather than antigenomic polarity. These are indicated in Figure 2 as RNAs 13 to 16 . As summarized in Figure 2, we tested nine different pairs of antigenomic RNAs and three pairs of genomic RNAs.

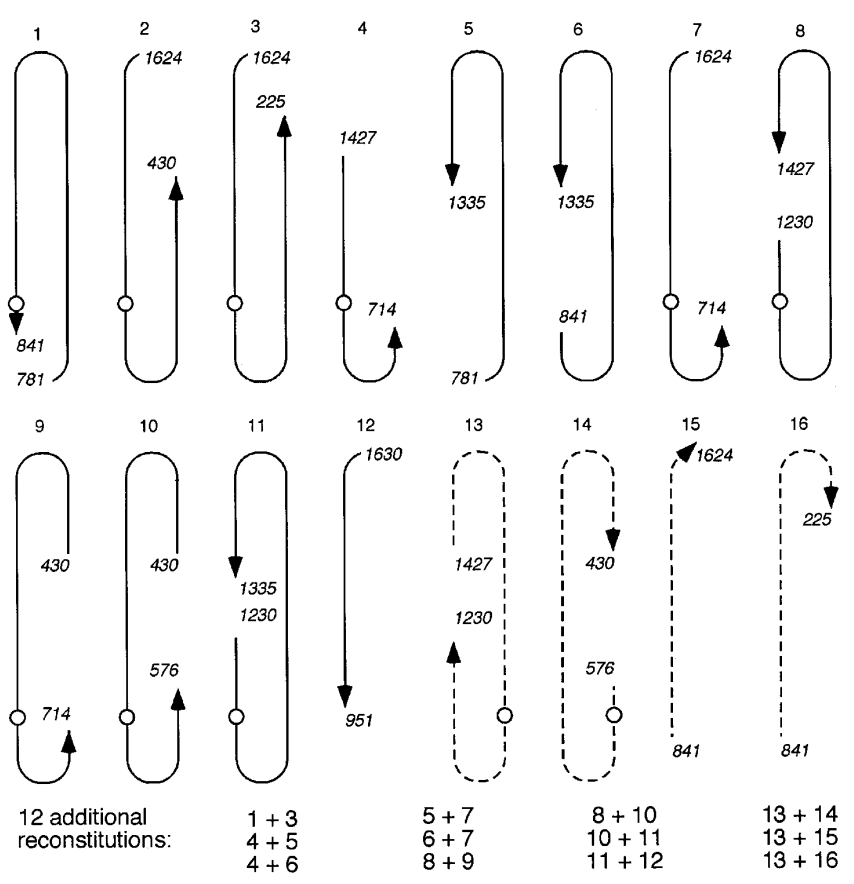

FIGURE 2. Additional examples of less than unit-length HDV RNAs used in reconstitution assays. The format is as in Figure 1A. Note that species 1-12 are antigenomic while 13-16 are genomic. The cleavage sites for antigenomic and genomic ribozymes are indicated by circles, corresponding to positions $901 / 900$ and $685 / 686$, respectively. At the bottom of the figure are shown 12 examples of pairs of RNA species that, after prior annealing, were transfected into $\delta 293$ cells and found to be capable of reconstituting HDV genome replication (Fig. 3A; data not shown). 
These RNAs and the combinations thereof were chosen to test the following variables: (1) antigenomic RNAs relative to genomic RNAs; (2) ability of a template RNA to fold intramolecularly (e.g., RNAs 8 and 13 have extensive rodlike folding, while RNAs 12 and 15 have none); (3) the predicted ability of the two partners, with or without a prior denaturation, to make an intermolecular hybrid using a rearrangement of the rodlike pairing (e.g., RNAs 10 and 11 have only $1335-1230=105 \mathrm{nt}$ available for interaction prior to denaturation, but afterward, the extent is $1679-$ $576+430-105=1428 \mathrm{nt}$ ); (4) the extent and location of the sequence overlap (e.g., for RNAs 4 and 5 the extent of the overlaps is reduced to $1427-1335=92$ and $841-$ $714=67$, while in contrast for RNAs 10 and 11 , the overlaps are much larger, $1230-576=654$ and $1679-1335+430=$ 774).

All of these 12 additional pairs achieved reconstitution. Northern data for five of these pairs are presented in Figure 3A. We observed that pairs of antigenomic or of genomic RNAs were both able to reconstitute. Overall, we observed some differences in the ability of the 12 pairs to achieve reconstitution (e.g., $5+7$ vs. $8+9$ in Fig. $3 \mathrm{~A}$ ), but we were unable to make clear correlations in terms of the effect of the predicted ability of the RNAs to fold intramolecularly, the ability to make intermolecular pairing, the extent of overlap in the intermolecular hybrid, or the location of these overlap regions. However, based on the total of 13 positive results obtained, we interpret that maybe any pair of RNAs (genomic or antigenomic) that (1) in theory can form an intermolecular rodlike structure after annealing, (2) encompass at least the full length of the HDV sequence,

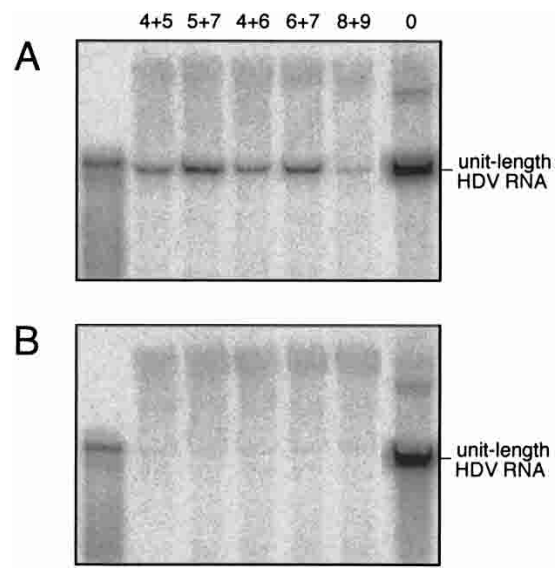

FIGURE 3. Reconstitution depends upon prior annealing of RNAs. (A) The reconstitution for five antigenomic RNA combinations as in Figure 1, with RNAs as described in Figure 2. The Northern analysis to detect genomic RNA was for total RNA taken at $4 \mathrm{~d}$ after transfection with pre-annealed antigenomic RNAs. (B) A parallel experiment except that the transfected RNAs were not pre-annealed. In each panel, the left lane is a standard of $1.1 \times$ unit-length HDV transcribed in vitro. At the right side is a positive control of RNA from cells transfected with RNA 0, which also provides a reference point for the migration position of unit-length HDV RNA. and (3) that have small direct sequence overlaps at the sites of discontinuity will be positive in this reconstitution assay.

Next we addressed the significance in our reconstitution assay of the prior denaturation and RNA annealing step. Parallel experiments were undertaken with RNAs that were not given the annealing prior to transfection. As shown for five RNA pairs in Figure 3B (and for all other combinations shown in Fig. 2 [data not shown]), Northern analyses no longer detected a band with the same mobility as unitlength HDV RNA. (The relatively faint band running somewhat slower than unit-length HDV is present even for RNA from untransfected cells and is considered to represent cross-hybridization to abundant cellular 18S rRNA [data not shown].) Thus, we conclude that the prior denaturation and annealing step was essential to achieve reconstitution.

In terms of this requirement for prior annealing, one of the successful pre-annealed pairs, RNAs $11+12$, has additional interest. RNA 12 was an RNA of the same size and sequence as the mRNA known to arise during normal HDV genome replication (Gudima et al. 2000). Moreover, this partner was transcribed so as to be like the natural mRNA, with a $5^{\prime}$-cap and a $3^{\prime}$-poly(A) sequence, and has been previously shown to be translated in vivo to yield the delta protein required to support HDV genome replication (Moraleda and Taylor 2001). This experiment was a positive control for the ability of a mRNA to interact with an RNA, such as RNA 11, and achieve reconstitution. However, this ability was lost when the two RNAs were not pre-annealed (data not shown). Further, when RNA 11 alone was transfected into $\delta 293$ cells efficiently expressing a mRNA for the delta protein, there was no reconstitution to produce a replication-competent genome (data not shown). The implications of this negative result are considered in the Discussion.

In the studies presented so far, all the transfections were achieved in 8293 , human embryonic kidney cells that conditionally express the small delta protein. We therefore examined whether reconstitution could also be achieved using Huh7, a human hepatocellular carcinoma cell line, using somewhat different experimental conditions. We mixed the annealed RNAs $13+14$ together with in vitro transcribed mRNA for the small delta protein. The latter was added as in the studies of Modahl et al. (1998). At the time of transfection the Huh7 cells contain none of the small delta protein that is essential for replication. Thus, it is only after translation of the cotransfected mRNA that there is an initial source of this delta protein. Again, Northern analyses of total RNA collected $4 \mathrm{~d}$ after transfection detected the accumulation of full-length HDV RNAs (data not shown), consistent with reconstitution also occurring under these conditions.

In summary, from these experiments we found that reconstitution could be achieved with both genomic and antigenomic RNAs. These RNAs could be of a range in primary sequence and predicted secondary structure. The ef- 


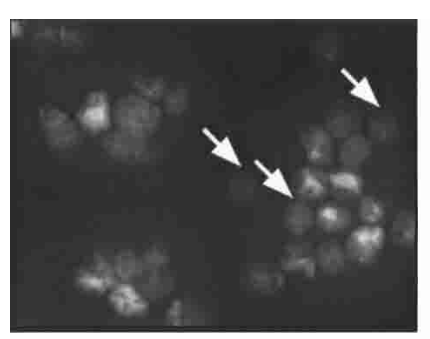

DAPI

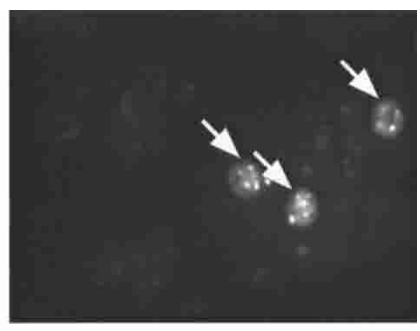

$\delta \mathrm{Ag}$

FIGURE 4. HDV reconstitution as assayed by immunocytochemistry. $\delta 293$ cells were transfected with a pre-annealed mix of RNAs $6+7$, as described in Figure 2. At day 3 of growth in the absence of tetracycline, the cells were fixed and stained with DAPI, followed by incubations with rabbit anti-delta protein antibody and Texas-red conjugated donkey anti-rabbit antibody. Microscopy was at $40 \times$. The arrows indicate cells interpreted as undergoing HDV replication.

ficiency of reconstitution was dependent upon the prior annealing of the RNAs before they were transfected. Finally, reconstitution could be achieved both in $\delta 293$ and with a somewhat modified strategy in Huh7 cells.

\section{Efficiency of reconstitution}

In all our reconstitution studies the efficiency was apparently low. That is, the accumulation of full-length HDV per average cell was at least 10 times less than that obtained for cells transfected with just a full-length linear HDV RNA. To better understand the basis for this inefficiency, we used three experimental strategies.

First, we took the total RNA from cells that had demonstrated reconstitution and used it to transfect new $\delta 293$ cells. At $5 \mathrm{~d}$ the total RNA was examined by Northern for unit-length HDV RNAs. This strategy routinely gave about a 12 -fold increase in the amount of HDV RNA per average cell (data not shown). Our initial interpretation was that the increase could have involved one or probably both of the following components. It could be that a greater fraction of cells was now successfully transfected. It could also be that the RNA used to initiate this transfection included HDV species that were unit-length circles, which we know are more efficient than linear RNAs at initiating replication (Gudima et al. 2004).

A second approach that was to directly quantitate HDV replication in individual cells using immunocytochemistry. For the $\delta 293$ cells we already knew that $100 \%$ are expressing the small delta protein (J. Chang, S.O. Gudima, C. Tarn, X. Nie, and J.M. Taylor, in prep.). When tetracycline is added to these cells the level of delta protein expression increases about 50-fold, and yet even in the absence of tetracycline, there is a sufficient amount of small delta protein expressed to allow the initiation of HDV replication following transfection with HDV RNA. Moreover, under the latter conditions, the replicating RNA soon produces its own mRNA for the small delta protein, leading to enhanced replication and enhanced levels of delta protein expressed per cell. Thus, with immunocytochemistry to detect the delta protein, we can quantitate those cells that are replicating the HDV genomes and making increased amounts of delta protein. An example of this strategy is shown in Figure 4, in which the cells were transfected with RNAs $6+7$. As expected, all cells show at least a low level of expression of delta protein. However, a small fraction of the cells show a much stronger signal. No such strong signals were detected in cells not transfected with HDV RNA (data not shown). Thus we interpret that these strong signals as indicative of increased amounts of delta protein produced during the replication of the HDV genome in these cells. This strong signal was intranuclear, nonnucleolar, and largely in nucleoplasmic specklelike structures. This distribution of the delta antigen is exactly as we have previously described as the characteristic pattern for HDV replication (Bichko and Taylor 1996). From a quantitation of these positive cells, we found that following transfection with a pair of annealed less than full-length HDV RNAs, $1.6 \%$ of the cells (10/620) went on to achieve HDV genome replication. When cells were transfected with unit-length linear RNA the percentage was higher, 9.4\% (94/998).

A third approach was used to test the efficiency of reconstitution in a somewhat different way. Previously we have reported the use of competition assays to determine the relative efficiency of two different HDV RNA templates to initiate genome replication (Gudima et al. 2004). In this strategy the two RNA species are cotransfected and so compete intracellularly. The two species differ at a small sequence-specific region and so are individually quantifiable, both before and after transfection, using Northern analyses and specific oligonucleotide probes. In this way we tested the initiation ability of a pair of annealed, less than fulllength RNAs, relative to a full-length linear RNA. As summarized in Table 1, we began with three different input ratios and then measured the output ratio at $5 \mathrm{~d}$ after transfection. Within the experimental errors we consider the three values of the output relative to the input ratio are

TABLE 1. Intermolecular competition assay to determine the relative efficiency of reconstitution

\begin{tabular}{|c|c|c|}
\hline \multicolumn{2}{|c|}{ Ratio of annealed RNAs $6+7$ to RNA 0} & \multirow{2}{*}{$\begin{array}{l}\text { Output ratio } \\
\text { expressed relative } \\
\text { to input ratio }\end{array}$} \\
\hline Input $($ day $=0)$ & Output (day = 5) & \\
\hline 10 & 0.15 & 0.015 \\
\hline 5 & 0.11 & 0.022 \\
\hline 1 & 0.079 & 0.079 \\
\hline
\end{tabular}

$\delta 293$ cells were transfected with RNA 0 and pre-annealed RNAs $6+7$. The molar ratio of RNAs $6+7$ to RNA 0 at input was determined from optical density measurements of gel-purified RNAs. At output, the ratio was determined by Northern analyses of extracted RNA assayed using sequential hybridization with specific oligonucleotide probes (Gudima et al. 2004). 
approximately the same. Thus, from this we deduce that the efficiency of initiation by reconstitution was $1.5 \%-8 \%$ relative to that for unit-length linear RNA.

In summary, from these three approaches we consider that the apparent low efficiency of reconstitution with annealed pairs of less than unit-length RNAs can primarily be explained as due to an early step. That is, the transfection procedure productively delivers the annealed RNAs to only a small fraction of the cells. A secondary reason may be that even when the annealed RNAs enter the cells, they are at a significant disadvantage for achieving initiation relative to even a unit-length linear RNA.

\section{DISCUSSION}

These studies demonstrated that following transfection of cells with a pair of HDV RNAs, each of which was less than full length, it was possible to achieve the replication of full-length RNAs, a phenomenon we refer to as "reconstitution". While traditional recombination assays involve at least one replication-competent RNA partner out of two, our approach employs a pair of less than full-length RNAs, neither of which was replication competent. Nevertheless, it is realized that what we define as genome reconstitution is actually a special form of viral RNA recombination (Lai 1992).

We consider it highly unlikely that this reconstitution was a consequence of changes made on the initial transfected RNA templates. In contrast, we consider that by template-switching or copy choice, during transcription, it is possible to achieve transcripts, some of which are greater than unit length, that can be subsequently processed to unit-length species and then act as templates for additional rounds of replication. We have previously shown that intramolecular template-switching can occur during the initiation of HDV replication from linear RNA templates (Chang and Taylor 2002; Gudima et al. 2004). Here, we show that it can occur intermolecularly, using as templates two different linear RNA molecules, each of which was less than full length. In addition, it was achieved in two different sources of human cells and under different experimental conditions.

For other RNA viruses, there are precedents for such reconstitution, and the mechanism is generally considered to be achieved via template-switching during transcription (Hajjou et al. 1996). In contrast, certain studies with poliovirus have been given a second interpretation, invoking restoration of the templates prior to transcription. Studies by Gmyl and coworkers report that if two less than full-length fragments of poliovirus RNA are pre-associated in vitro and then transfected, there can be achieved the reconstitution of plaque-forming virus. These authors interpret this rescue as a consequence of some forms of processing, including ligation, of the input RNA fragments (Gmyl et al. 1999, 2003). For the following reasons, we consider this second type of interpretation as inconsistent with our observations. First, some of the HDV template RNAs used in our study had either a missing or an incomplete ribozyme domain (Fig. 2). This excludes the possibility for cleavage/ligation of input RNAs. Second, we have previously shown that linear HDV RNA templates are not detectably "healed" prior to transcription (Chang and Taylor 2002). Third, our recent study comparing the initiation abilities of different HDV RNAs has shown that certain input linear RNA templates that were of at least unit length and yet incapable of undergoing ribozyme-mediated processing were nevertheless able to initiate genome replication (Gudima et al. 2004). Fourth, unlike in the studies with poliovirus and a pestivirus, the replication of HDV RNA depends upon a preexisting hostencoded polymerase. In summary, these studies support our interpretation of direct utilization, without any prior processing, of the transfected HDV RNAs as templates by the transcription machinery, which via template-switching leads to the observed reconstitution of full-length HDV RNA.

Almost all the available evidence indicates that host pol II is the enzyme involved in HDV RNA-directed transcription (Modahl et al. 2000; Moraleda and Taylor 2001; Macnaughton et al. 2002; Taylor 2003). Therefore, the reconstitution results presented in this study suggest that RNA polymerase II is able to make correct intermolecular template-switching during RNA-directed RNA synthesis. For both inter- and intramolecular events we expect there may be a significant fraction of template-switching events that are nonproductive. In addition, we observed that the efficiency of intermolecular reconstitution was at least 12 times less than for intramolecular template-switching (Table 1). One possible explanation for this reduction is that the number of productive template switches leading to replication-competent RNAs synthesis is lower when switching occurs between two different template molecules.

For our studies the cells are initially transfected with an average of one million molecules of HDV RNA per cell, although we do not know how many of these actually are appropriately delivered to a site in the nucleus for the initiation of transcription by the host polymerase. Next, for these transfected pairs of annealed RNAs, there may then be many template switches, most of which are nonproductive, but with a rare replication-competent winner. The chance of creating a winner will no doubt depend on the locations of the transcription start site and the position(s) used for the switching. Furthermore, the RNA polymerase in some cases might be required to make not one, but two correct intermolecular jumps in order to produce replicating RNA. Apparently, the rate for intermolecular template-switching is less efficient than for intramolecular. This interpretation is consistent with our observation that a smaller fraction of cells was able to initiate replication from two shorter than unit-length RNAs than from a single linear unit-length RNA. 
While it is generally agreed that the HDV genome and mRNA are synthesized by RNA polymerase II, there is the claim that antigenomic RNA is transcribed by a different polymerase, possibly RNA polymerase I (Modahl et al. 2000; Macnaughton et al. 2002). However, we consider our observation that in vivo reconstitution was equally successful with pairs of either genomic or antigenomic RNA to be more consistent with the interpretation that genomic and antigenomic HDV RNAs in vivo are actually transcribed by the same host RNA polymerase. However, one might hypothesize that pol II and pol I can both perform RNAdirected RNA transcription and, furthermore, both can carry out template-switching with essentially equal efficiencies.

We found that for efficient reconstitution the input RNAs needed to be pre-annealed in vitro; no detectable reconstitution was achieved when annealing was omitted (Fig. 3B). In particular, consider again the example of RNAs $11+12$. RNA 12 was designed to be similar to the mRNA for the delta protein. It has the same length and has both a $5^{\prime}$-cap and a $3^{\prime}$-poly(A). When this RNA was annealed with RNA 11 and then transfected, reconstitution was achieved. Without prior annealing there was no reconstitution. Furthermore, RNA 11 was not able to achieve reconstitution with the delta antigen mRNA already present in the transfected cells. While several interpretations could be given for this result, our negative findings do not support the speculation arising from a previous study that HDV might have arisen during transcription via interaction between a hypothetical mammalian viroid and a host mRNA (Brazas and Ganem 1996; Robertson 1996).

This leads to a discussion of whether HDV RNAs undergo traditional recombination involving at least one replication-competent partner in addition to the special form of recombination that we have defined as reconstitution. We have made various unsuccessful attempts to achieve such traditional recombination. In studies with two replicating RNAs, no recombination was detected (T.-T. Wu and J.M. Taylor, and S. Gudima and J.M. Taylor, data not shown). In yet another study, with a series of five different less than unit-length RNAs transfected into cells already replicating $\mathrm{HDV}$, no recombination was detected, and yet if a less than unit-length RNA was annealed to a linear unitlength RNA, prior to transfection, recombination was achieved (data not shown). From these studies we consider that (in the absence of prior annealing in vitro) recombination for HDV in vivo either does not happen or occurs at a low frequency. This interpretation is in contrast to one claim of recombination occurring in patients infected with more than one HDV genotype (Wu et al. 1999). It is also in contrast to claims for recombination between plant viroids (Koltunow and Rezaian 1989; Rezaian 1990). Nevertheless, it remains possible that in the presence of some form of selective pressure, recombination of HDV RNAs might be demonstrated.
We observed RNA reconstitution with many different combinations of less than full-length RNAs (Figs. 1-3). Since each of these RNAs lacked different portions of the rodlike structure it would seem that there were many acceptable locations for the intermolecular template-switching by RNA polymerase II. We found that successful reconstitutions depended upon prior annealing of the RNAs. While many different structures might have been achieved in this annealing, we favor the interpretation that somehow the rodlike folding ability of HDV RNAs, unique relative to other RNA viruses, was central not only in the necessary intermolecular association of the RNAs but also as a major facilitator of the subsequent template-switching event(s) that occur in the transfected cell. In addition, it might be that the binding of the delta protein which we know is specific for such rodlike folding (Chao et al. 1991) somehow contributes to the template-switching leading to successful reconstitution.

Overall, our studies provide the first evidence for in vivo intermolecular template-switching during RNA-directed RNA synthesis as catalyzed possibly by host RNA polymerase II. This gives new insights into unrecognized capabilities of this enzyme. When it acts "normally" on templates that are DNA, this multisubunit enzyme is known to exist in at least three forms (IIA, II0, and possibly IIB) and to make associations with many host factors. Some of the details of this transcription have been incorporated into models that make use of crystal structures of the polymerase, both with and without associated factors and template molecules (Cramer et al. 2000, 2001; Gnatt et al. 2001; Davis et al. 2002; Bushnell and Kornberg 2003; Bushnell et al. 2004; Westover et al. 2004). The challenge now is to determine to what extent such studies based on transcription from DNA templates can be extrapolated to the same enzyme when it acts in vivo to not only copy templates that are RNA but also to carry out template-switching that can be both intraand intermolecular. A current limitation is that, so far, no one has achieved a robust system for HDV RNA-directed transcription in vitro (Beard et al. 1996; Filipovska and Konarska 2000; Gudima et al. 2000; Moraleda and Taylor 2001; Yamaguchi et al. 2001). As an alternative approach, HDV RNA-directed transcription is currently being studied using immuno-precipitation of transcription complexes that have been formed in vivo (X. Nie, J. Chang, and J.M. Taylor, unpubl.). In addition, future in vivo studies using the reconstitution assay described here may provide a strategy to define what is actually "recognized" by the RNA polymerase to begin RNA-directed transcription.

\section{MATERIALS AND METHODS}

\section{Cell culture and transfection}

Huh7, a human hepatocellular carcinoma cell line (Nakabayashi et al. 1982), was cultured, using Dulbecco's modified Eagle's medium (DMEM), supplemented with $10 \%$ fetal bovine serum in the ab- 
sence of antibiotics. $\delta 293$, a human embryonic kidney cell line expressing small delta antigen under conditional control of tetracycline, was as described previously (J. Chang, S.O. Gudima, C. Tarn, X. Nie, and J.M. Taylor, in prep.; Gudima et al. 2004). These cells were cultured in DMEM in the presence of hygromycin B (Invitrogen) $(200 \mu \mathrm{g} / \mathrm{mL})$ and blasticidin S HCl (Invitrogen) $(15 \mu \mathrm{g} / \mathrm{mL})$. Transfection of either $\delta 293$ or Huh7 cells with RNA was performed as previously described (Gudima et al. 2004) using Lipofectamine 2000 (Invitrogen) as recommended by the manufacturer.

\section{RNA preparation and annealing of RNAs}

The strategy for constructing HDV vectors for in vitro RNA transcription was as previously described (Gudima et al. 2004). Plasmids bearing NheI-NheI and EcoNI-EcoNI HDV-specific monomeric insertions were similarly constructed. All constructs have a T7 promoter so that RNAs of desired size can be transcribed after linearization of the plasmids with appropriate restriction enzymes. In vitro transcription and subsequent RNA purification were as described previously (Gudima et al. 1997, 2004). RNA transcripts used in this study are described in Figures 1 and 2. For the intermolecular competition study presented in Table 1, genetically marked RNA was as in Gudima et al. (2004). mRNA encoding the small delta antigen was prepared as described previously (Moraleda and Taylor 2001). Shorter than unit-length HDV RNAs in equimolar amounts were pre-annealed in a buffer of $50 \mathrm{mM}$ Tris (pH 7.5) and $100 \mathrm{mM} \mathrm{NaCl}$ by boiling for $2 \mathrm{~min}$ and then slow cooling to room temperature.

\section{Northern analyses}

Total RNA was extracted using Tri Reagent (Molecular Research Center). Obtained RNA samples were glyoxalated and assayed by Northern analysis using gels of $1.7 \%$ agarose, as previously described (Kuo et al. 1989; Gudima et al. 2002). For Table 1, instead of using labeled RNA probes we used end-labeled sequence-specific oligonucleotides, as described previously (Gudima et al. 2004).

\section{Immunofluorescence}

The procedure was similar to one described previously (Bichko and Taylor 1996). 8293 cells, nontransfected or transfected with pre-annealed either two shorter than unit-length HDV RNAs $(6+7$ as described in Fig. 2) or linear monomer, were further cultured in the absence of tetracycline. At day 2, cells were reseeded at a lower density into glass slide chambers (Nalge Nunc International). At day 3, the fixation and permeabilization of monolayer cultures was performed with $4.0 \%$ paraformaldehyde in phosphate-buffered saline (PBS) containing $0.1 \%$ Triton $\mathrm{X}-100$ (PBST) for $15 \mathrm{~min}$ at room temperature. Cells were then washed twice with PBS, blocked with $3 \%$ bovine serum albumin (BSA) in PBST for $30 \mathrm{~min}$ at room temperature, incubated with a 1:1000 dilution in $\mathrm{PBST} / 1 \%$ BSA of rabbit polyclonal antibodies against delta protein (Bichko and Taylor 1996) for $1 \mathrm{~h}$ at $37^{\circ} \mathrm{C}$, washed three times with $\mathrm{PBST} / 1 \% \mathrm{BSA}$, and finally incubated with a $1: 500$ dilution of secondary donkey-anti-rabbit antibody labeled with rhodamine (Jackson ImmunoResearch) together with 4',6-diamidino-2-phenylin-dole (DAPI; Sigma) (at $1 \mu \mathrm{g} / \mathrm{mL}$ ) to stain cellular DNA. Mounted samples were analyzed with an Inverted Nikon TE2000-U microscope using a 40× Plan Fluor ELWD, WD 3.7-2.7 objective and specific filter blocks, equipped with a Cascade 650 (Photometrics) monochrome camera and running MetaVue (Universal Imaging) software for acquisition. Images were processed with Adobe Photoshop 7.0 and Canvas 9.0 software.

\section{ACKNOWLEDGMENTS}

This work was supported by NIH grants AI-26522 and CA-06927 and by an appropriation from the Commonwealth of Pennsylvania. Critical comments on the manuscript were given by Glenn Rall and Richard Katz. We thank Irina Shchaveleva and the Fox Chase Cancer Center Imaging Facility (Sandra Jablonski, Manager) for help with the immunofluorescence studies.

Received August 20, 2004; accepted October 11, 2004.

\section{REFERENCES}

Beard, M.R., Macnaughton, T.B., and Gowans EJ. 1996. Identification and characterization of a hepatitis delta virus RNA transcriptional promoter. J. Virol. 70: 4986-4995.

Bergmann, M., Garcia-Sastre, A., and Palese, P. 1992. Transfectionmediated recombination of influenza A virus. J. Virol. 66: 75767580.

Bichko, V.V. and Taylor, J.M. 1996. Redistribution of the delta antigens in cells replicating the genome of hepatitis delta virus. J. Virol. 70: 8064-8070.

Brazas, R. and Ganem, D. 1996. A cellular homolog of hepatitis delta antigen: Implications for viral replication and evolution. Science 274: 90-94.

Bushnell, D.A. and Kornberg, R.D. 2003. Complete, 12-subunit RNA polymerase II at 4.1- $\AA$ resolution: Implications for the initiation of transcription. Proc. Natl. Acad. Sci. 100: 6969-6973.

Bushnell, D.A., Westover, K.D., Davis, R.E., and Kornberg, R.D. 2004. Structural basis of transcription: An RNA polymerase II-TFIIB cocrystal at 4.5 Angstroms. Science 303: 983-988.

Chang, J. and Taylor, J. 2002. In vivo RNA-directed transcription, with template switching, by a mammalian RNA polymerase. EMBO J. 21: 157-164.

Chao, M., Hsieh, S.-Y., and Taylor, J. 1990. Role of two forms of the hepatitis delta virus antigen: Evidence for a mechanism of selflimiting genome replication. J. Virol. 64: 5066-5069.

. 1991. The antigen of hepatitis delta virus: Examination of in vitro RNA-binding specificity. J. Virol. 65: 4057-4062.

Chare, E.R., Gould, E.A., and Holmes, E.C. 2003. Phylogenetic analysis reveals a low rate of homologous recombination in negative-sense RNA viruses. J. Gen. Virol. 84: 2691-2703.

Chen, P.-J., Kalpana, G., Goldberg, J., Mason, W., Werner, B., Gerin, J., and Taylor, J. 1986. Structure and replication of the genome of hepatitis delta virus. Proc. Natl. Acad. Sci. 83: 8774-8778.

Chetverin, A.B. 1999. The puzzle of RNA recombination. FEBS Lett. 460: $1-5$.

Cramer, P., Bushnell, D.A., Fu, J., Gnatt, A.L., Maier-Davis, B., Thompson, N.E., Burgess, R.R., Edwards, A.M., David, P.R., and Kornberg, R.D. 2000. Architecture of RNA polymerase II and implications for the transcription mechanism. Science 288: 640-649.

Cramer, P., Bushnell, D.A., and Kornberg, R.D. 2001. Structural basis of transcription: RNA polymerase II at $2.8 \AA$ resolution. Science 292: 1863-1876.

Davis, J.A., Takagi, Y., Kornberg, R.D., and Asturias, F.A. 2002. Structure of the yeast RNA polymerase II holoenzyme: Mediator con- 
formation and polymerase interaction. Mol. Cell 10: 409-415.

Filipovska, J. and Konarska, M.M. 2000. Specific HDV RNA-templated transcription by pol II in vitro. RNA 6: 41-54.

Flint, S.J., Enquist, L.W., Racaniello, V.R., and Skalka, A.M. 2004. Principles of virology: Molecular biology, pathogenesis, and control of animal viruses. ASM Press, Washington, DC.

Gallei, A., Pankraz, A., Thiel, H.J., and Becher, P. 2004. RNA recombination in vivo in the absence of viral replication. J. Virol. 78: 6271-6281.

Gerin, J.L., Casey, J.L., and Purcell, R.H. 2001. Hepatitis delta virus. In Fields' Virology (eds. D.M. Knipe and P.M. Howley), pp. 30373050. Lippincott Williams \& Wilkins, Philadelphia, PA.

Gmyl, A.P., Belousov, E.V., Maslova, S.V., Khitrina, E.V., Chetverin, A.B., and Agol, V.I. 1999. Nonreplicative RNA recombination in poliovirus. J. Virol. 73: 8958-8965.

Gmyl, A.P., Korshenko, S.A., Belousov, E.V., Khitrina, E.V., and Agol, V.I. 2003. Nonreplicative homologous RNA recombination: Promiscuous joining of RNA pieces? RNA 9: 1221-1231.

Gnatt, A.L., Cramer, P., Fu, J., Bushnell, D.A., and Kornberg, R.D. 2001. Structural basis of transcription: An RNA polymerase II elongation complex at $3.3 \AA$ resolution. Science 292: 1876-1882.

Gudima, S.O., Kazantseva, E.G., Kostyuk, D.A., Shchaveleva, I.L., Grishchenko, O.I., Memelova, L.V., and Kochetkov, S.N. 1997. Deoxyribonucleotide-containing RNAs: A novel class of templates for HIV-1 reverse transcriptase. Nucleic Acids Res. 25: 4614-4618.

Gudima, S., Wu, S.-Y., Chiang, C.-M., Moraleda, G., and Taylor, J. 2000. Origin of the hepatitis delta virus mRNA. J. Virol. 74: 72047210.

Gudima, S.O., Chang, J., Moraleda, G., Azvolinsky, A., and Taylor, J. 2002. Parameters of human hepatitis delta virus replication: The quantity, quality, and intracellular distribution of viral proteins and RNA. J. Virol. 76: 3709-3719.

Gudima, S.O., Chang, J., and Taylor, J.M. 2004. Features affecting the ability of hepatitis delta virus RNAs to initiate RNA-directed RNA synthesis. J. Virol. 78: $5737-5744$.

Hajjou, M., Hill, K.R., Subramaniam, S.V., Hu, J.Y., and Raju, R. 1996. Nonhomologous RNA-RNA recombination events at the $3^{\prime}$ nontranslated region of the Sindbis virus genome: Hot spots and utilization of nonviral sequences. J. Virol. 70: 5153-5164.

Koetzner, C.A., Parker, M.M., Ricard, C.S., Sturman, L.S., and Masters, P.S. 1992. Repair and mutagenesis of the genome of a deletion mutant of the coronavirus mouse hepatitis virus by targeted RNA recombination. J. Virol. 66: 1841-1848.

Koltunow, A.M. and Rezaian, M.A. 1989. Grapevine viroid 1B, a new member of the apple scar skin viroid group contains the left terminal region of tomato planta macho viroid. Virology 170: 575-578.

Kos, A., Dijkema, R., Arnberg, A.C., van der Meide, P.H., and Schellekens, H. 1986. The hepatitis delta $(\delta)$ virus possesses a circular RNA. Nature 323: 558-560.

Kuo, M.Y.-P., Chao, M., and Taylor, J. 1989. Initiation of replication of the human hepatitis delta virus genome from cloned DNA: Role of delta antigen. J. Virol. 63: 1945-1950.

Lai, M.M.C. 1992. Genetic recombination in RNA viruses. Curr. Topics
Microbiol. Immunol. 176: 21-32.

Lazinski, D.W. and Taylor, J.M. 1994. Expression of hepatitis delta virus RNA deletions: cis and trans requirements for self-cleavage, ligation, and RNA packaging. J. Virol. 68: 2879-2888.

Liao, C.L. and Lai, M.M. 1992. RNA recombination in a coronavirus: Recombination between viral genomic RNA and transfected RNA fragments. J. Virol. 66: 6117-6124.

Macnaughton, T.B., Shi, S.T., Modahl, L.E., and Lai, M.M. 2002. Rolling circle replication of hepatitis delta virus RNA is carried out by two different cellular RNA polymerases. J. Virol. 76: 3920-3927.

Modahl, L.E. and Lai, M.M. 1998. Transcription of hepatitis delta antigen mRNA continues throughout hepatitis delta virus (HDV) replication: A new model of HDV transcription and replication. J. Virol. 72: 5449-5456.

Modahl, L.E., Macnaughton, T.B., Zhu, N., Johnson, D.L., and Lai, M.M.C. 2000. RNA-dependent replication and transcription of hepatitis delta virus RNA involve distinct cellular RNA polymerases. Mol. Cell. Biol. 20: 6030-6039.

Moraleda, G. and Taylor, J. 2001. Host RNA polymerase requirements for transcription of the human hepatitis delta virus genome. J. Virol. 75: 10161-10169.

Nagy, P.D. and Simon, A.E. 1997. New insights into the mechanisms of RNA recombination. Virology 235: 1-9.

Nakabayashi, H., Taketa, K., Miyano, K., Yamane, T., and Sato, J. 1982. Growth of human hepatoma cell lines with differentiated functions in chemically defined medium. Cancer Res. 42: 38583863.

Rezaian, M.A. 1990. Australian grapevine viroid-Evidence for extensive recombination between viroids. Nucleic Acids Res. 18: 18131818.

Robertson, H.D. 1996. How did replicating and coding RNAs first get together? Science 274: 66-67.

Spann, K.M., Collins, P.L., and Teng, M.N. 2003. Genetic recombination during coinfection of two mutants of human respiratory syncytial virus. J. Virol. 77: 11201-11211.

Taylor, J.M. 2003. Replication of human hepatitis delta virus: Recent developments. Trends Microbiol. 11: 185-190.

Wang, K.-S., Choo, Q.-L., Weiner, A.J., Ou, J.-H., Najarian, C., Thayer, R.M., Mullenbach, G.T., Denniston, K.J., Gerin, J.L., and Houghton, M. 1986. Structure, sequence and expression of the hepatitis delta viral genome. Nature 323: 508-513.

Westover, K.D., Bushnell, D.A., and Kornberg, R.D. 2004. Structural basis of transcription: Separation of RNA from DNA by RNA polymerase II. Science 303: 1014-1016.

Worobey, M. and Holmes, E.C. 1999. Evolutionary aspects of recombination in RNA viruses. J. Gen. Virol. 80: 2535-2543.

Wu, J.C., Chiang, T.Y., Shiue, W.K., Wang, S.Y., Sheen, I.J., Huang, Y.H., and Syu, W.J. 1999. Recombination of hepatitis D virus RNA sequences and its implications. Mol. Biol. Evol. 16: 1622-1632.

Yamaguchi, Y., Filipovska, J., Yano, K., Furuya, A., Inukai, N., Narita, T., Wada, T., Sugimoto, S., Konarska, M.M., and Handa, H. 2001. Stimulation of RNA polymerase II elongation by hepatitis delta antigen. Science 293: 124-127. 

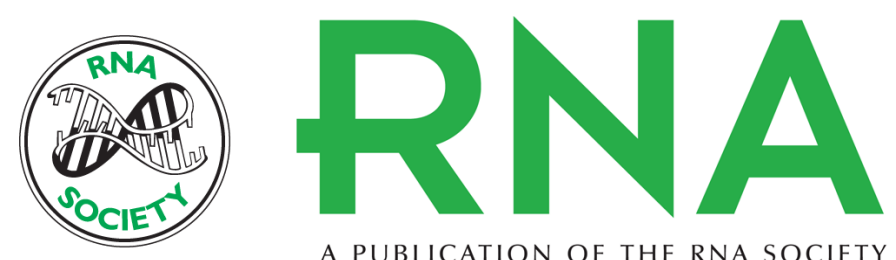

A PUBLICATION OF THE RNA SOCIETY

\title{
Reconstitution in cultured cells of replicating HDV RNA from pairs of less than full-length RNAs
}

\author{
SEVERIN O. GUDIMA, JINHONG CHANG and JOHN M. TAYLOR
}

RNA 2005 11: 90-98

References This article cites 47 articles, 33 of which can be accessed free at: http://rnajournal.cshlp.org/content/11/1/90.full.html\#ref-list-1

\section{License}

Email Alerting Service

Receive free email alerts when new articles cite this article - sign up in the box at the top right corner of the article or click here. 